\title{
Identifying 1950s American Jazz Musicians: Fine-Grained IsA Extraction via Modifier Composition
}

\author{
Ellie Pavlick* \\ University of Pennsylvania \\ 3330 Walnut Street \\ Philadelphia, Pennsylvania 19104 \\ epavlick@seas. upenn.edu
}

\author{
Marius Paşca \\ Google Inc. \\ 1600 Amphitheatre Parkway \\ Mountain View, California 94043 \\ mars@google.com
}

\begin{abstract}
We present a method for populating fine-grained classes (e.g., "1950s American jazz musicians") with instances (e.g., Charles Mingus). While stateof-the-art methods tend to treat class labels as single lexical units, the proposed method considers each of the individual modifiers in the class label relative to the head. An evaluation on the task of reconstructing Wikipedia category pages demonstrates a $>10$ point increase in AUC, over a strong baseline relying on widely-used Hearst patterns.
\end{abstract}

\section{Introduction}

The majority of approaches (Snow et al., 2006; Shwartz et al., 2016) for extracting IsA relations from text rely on lexical patterns as the primary signal of whether an instance belongs to a class. For example, observing a pattern like " $X$ such as $Y$ " is a strong indication that $Y$ (e.g., "Charles Mingus") is an instance of class X (e.g., "musician") (Hearst, 1992).

Methods based on these "Hearst patterns" assume that class labels can be treated as atomic lexicalized units. This assumption has several significant weakness. First, in order to recognize an instance of a class, these patternbased methods require that the entire class label be observed verbatim in text. The requirement is reasonable for class labels containing a single word, but in practice, there are many possible fine-grained classes: not only " $m u$ sicians" but also "1950s American jazz musicians". The probability that a given label will appear in its entirety within one of the expected patterns is very low, even in large

\footnotetext{
${ }^{*}$ Contributed during an internship at Google.
}

\begin{tabular}{|c|}
\hline$\underline{1950 s}$ American jazz musicians \\
\hline $\begin{array}{l}\text {... seminal musicians such as Charles Mingus } \\
\text { and George Russell... }\end{array}$ \\
\hline $\begin{array}{l}\text {... A virtuoso bassist and composer, Mingus ir- } \\
\text { revocably changed the face of jazz... }\end{array}$ \\
\hline $\begin{array}{l}\text {... Mingus truly was a product of America in } \\
\text { all its historic complexities... }\end{array}$ \\
\hline $\begin{array}{l}\text {... Mingus dominated the scene back in the } \\
\text { 1950s and 1960s... }\end{array}$ \\
\hline
\end{tabular}

Figure 1: We extract instances of fine-grained classes by considering each of the modifiers in the class label individually. This allows us to extract instances even when the full class label never appears in text.

amounts of text. Second, when class labels are treated as though they cannot be decomposed, every class label must be modeled independently, even those containing overlapping words ("American jazz musician", "French jazz musician"). As a result, the number of meaning representations to be learned is exponential in the length of the class label, and quickly becomes intractable. Thus, compositional models of taxonomic relations are necessary for better language understanding.

We introduce a compositional approach for reasoning about fine-grained class labels. Our approach is based on the notion from formal semantics, in which modifiers ( "1950s") correspond to properties that differentiate instances of a subclass ("1950s musicians") from instances of the superclass ("musicians") (Heim and Kratzer, 1998). Our method consists of two stages: interpreting each modifier relative to the head ("musicians active during 1950s"), and using the interpretations to identify instances of the class from text (Figure 1). Our main contributions are: 1) a compositional method for IsA extraction, which in- 
volves a novel application of noun-phrase paraphrasing methods to the task of semantic taxonomy induction and 2) the operationalization of a formal semantics framework to address two aspects of semantics that are often kept separate in NLP: assigning intrinsic "meaning" to a phrase, and reasoning about that phrase in a truth-theoretic context.

\section{Related Work}

Noun Phrase Interpretation. Compound noun phrases ("jazz musician") communicate implicit semantic relations between modifiers and the head. Many efforts to provide semantic interpretations of such phrases rely on matching the compound to pre-defined patterns or semantic ontologies (Fares et al., 2015; Ó Séaghdha and Copestake, 2007; Tratz and Hovy, 2010; Surtani and Paul, 2015; Choi et al., 2015). Recently, interpretations may take the form of arbitrary natural language predicates (Hendrickx et al., 2013). Most approaches are supervised, comparing unseen noun compounds to the most similar phrase seen in training (Wijaya and Gianfortoni, 2011; Nulty and Costello, 2013; Van de Cruys et al., 2013). Other unsupervised approaches apply information extraction techniques to paraphrase noun compounds (Kim and Nakov, 2011; Xavier and Strube de Lima, 2014; Paşca, 2015). They focus exclusively on providing good paraphrases for an input noun compound. To our knowledge, ours is the first attempt to use these interpretations for the downstream task of IsA relation extraction.

IsA Relation Extraction. Most efforts to acquire taxonomic relations from text build on the seminal work of Hearst (1992), which observes that certain textual patterns-e.g., " $X$ and other $Y$ "-are high-precision indicators of whether $X$ is a member of class $Y$. Recent work focuses on learning such patterns automatically from corpora (Snow et al., 2006; Shwartz et al., 2016). These IsA extraction techniques provide a key step for the more general task of knowledge base population. The "universal schema" approach (Riedel et al., 2013; Kirschnick et al., 2016; Verga et al., 2017), which infers relations using matrix factorization, often includes Hearst patterns as input features. Graphical (Bansal et al., 2014) and joint inference models (Movshovitz-Attias and Cohen, 2015) typically require Hearst patterns to define an inventory of possible classes. A separate line of work avoids Hearst patterns by instead exploiting semi-structured data from HTML markup (Wang and Cohen, 2009; Dalvi et al., 2012; Pasupat and Liang, 2014). These approaches all share the limitation that, in practice, in order for a class to be populated with instances, the entire class label has to have been observed verbatim in text. This requirement limits the ability to handle arbitrarily fine-grained classes. Our work addresses this limitation by modeling fine-grained class labels compositionally. Thus the proposed method can combine evidence from multiple sentences, and can perform IsA extraction without requiring any example instances of a given class. ${ }^{1}$

Taxonomy Construction. Previous work on the construction of a taxonomy of IsA relations (Flati et al., 2014; de Melo and Weikum, 2010; Kozareva and Hovy, 2010; Ponzetto and Strube, 2007; Ponzetto and Navigli, 2009) considers that task to be different than extracting a flat set of IsA relations from text in practice. Challenges specific to taxonomy construction include overall concept positioning and how to discover whether concepts are unrelated, subordinated or parallel to each other (Kozareva and Hovy, 2010); the need to refine and enrich the taxonomy (Flati et al., 2014); the difficulty in adding relevant IsA relations towards the top of the taxonomy (Ponzetto and Navigli, 2009); eliminating cycles and inconsistencies (Ponzetto and Navigli, 2009; Kozareva and Hovy, 2010). For practical purposes, these challenges are irrelevant when extracting flat IsA relations. Whereas Flati et al. (2014); Bizer et al. (2009); de Melo and Weikum (2010); Nastase and Strube (2013); Ponzetto and Strube (2007); Ponzetto and Navigli (2009); Hoffart et al. (2013) rely on data within human-curated resources, our work operates over unstructured text. Resources constructed in Bizer et al. (2009); Nastase and Strube (2013); Hoffart et al. (2013) contain not just a taxonomy of IsA relations,

\footnotetext{
${ }^{1}$ Pasupat and Liang (2014) also focuses on zero-shot IsA extraction, but exploits HTML document structure, rather than reasoning compositionally.
} 
but also relation types other than IsA.

\section{Modifiers as Functions}

Formalization. In formal semantics, modification is modeled as function application. Specifically, let $M H$ be a class label consisting of a head $H$, which we assume to be a common noun, preceded by a modifier $M$. We use 『·』 to represent the "interpretation function" that maps a linguistic expression to its denotation in the world. The interpretation of a common noun is the set of entities ${ }^{2}$ in the universe $\mathcal{U}$. They are denoted by the noun (Heim and Kratzer, 1998):

$$
\llbracket H \rrbracket=\{e \in \mathcal{U} \mid e \text { is a } H\}
$$

The interpretation of a modifier $M$ is a function that maps between sets of entities. That is, modifiers select a subset ${ }^{3}$ of the input set:

$$
\llbracket M \rrbracket(H)=\{e \in H \mid e \text { satisfies } M\}
$$

This formalization leaves open how one decides whether or not "e satisfies $M$ ". This nontrivial, as the meaning of a modifier can vary depending on the class it is modifying: if $e$ is a "good student", $e$ is not necessarily a "good person", making it difficult to model whether "e satisfies good" in general. We therefore reframe the above equation, so that the decision of whether " $e$ satisfies $M$ " is made by calling a binary function $\phi_{M}$, parameterized by the class $H$ within which $e$ is being considered:

$$
\llbracket M \rrbracket(H)=\left\{e \in H \mid \phi_{M}(H, e)\right\}
$$

Conceptually, $\phi_{M}$ captures the core "meaning" of the modifier $M$, which is the set of properties that differentiate members of the output class $M H$ from members of the more general input class $H$. This formal semantics framework has two important consequences. First, the modifier has an intrinsic "meaning". The properties entailed by the modifier are independent of the particular state of the world. This makes it possible to make inferences about "1950s musician" even if no

\footnotetext{
${ }^{2}$ We use "entities" and "instances" interchangeably; "entities" is standard terminology in linguistics.

${ }^{3}$ As does virtually all previous work in information extraction, we assume that modifiers are subsective, acknowledging the limitations (Kamp and Partee, 1995).
}

1950s musician have been observed. Second, the modifier is a function that can be applied in a truth-theoretic setting. That is, applying "1950s" to the set of "musicians" returns exactly the set of "1950s musicians".

Computational Approaches. While the notion of modifiers as functions has been incorporated into computational models previously, prior work focuses on either assigning an intrinsic meaning to $M$ or on operationalizing $M$ in a truth-theoretic sense, but not on doing both simultaneously. For example, Young et al. (2014) focuses exclusively on the subset selection aspect of modification. That is, given a set of instances $H$ and a modifier $M$, their method could return the subset $M H$. However, their method does not model the meaning of the modifier itself, so that, e.g., if there were no red cars in their model of the world, the phrase "red cars" would have no meaning. In contrast, Baroni and Zamparelli (2010) models the meaning of modifiers explicitly as functions that map between vector-space representations of nouns. However, their model focuses on similarity between class labels-e.g., to say that "important routes" is similar to "major roads"-and it is not obvious how the method could be operationalized in order to identify instances of those classes. A contribution of our work is to model the semantics of $M$ intrinsically, but in a way that permits application in the model theoretic setting. We learn an explicit model of the "meaning" of a modifier $M$ relative to a head $H$, represented as a distribution over properties that differentiate the members of the class $M H$ from those of the class $H$. We then use this representation to identify the subset of instances of $H$, which constitute the subclass $M H$.

\section{Learning Modifier Interpretations}

\subsection{Setup}

For each modifier $M$, we would like to learn the function $\phi_{M}$ from Eq. 3. Doing so makes it possible, given $H$ and an instance $e \in H$, to decide whether $e$ has the properties required to be an instance of $M H$. In general, there is no systematic way to determine the implied relation between $M$ and $H$, as modifiers can arguably express any semantic relation, given the right context (Weiskopf, 
2007). We therefore model the semantic relation between $M$ and $H$ as a distribution over properties that could potentially define the subclass $M H \subseteq H$. We will refer to this distribution as a "property profile" for $M$ relative to $H$. We make the assumption that relations between $M$ and $H$ that are discussed more often are more likely to capture the important properties of the subclass $M H$. This assumption is not perfect (Section 4.4) but has given good results for paraphrasing noun phrases (Nakov and Hearst, 2013; Paşca, 2015). Our method for learning property profiles is based on the unsupervised method proposed by Paşca (2015), which uses query logs as a source of common sense knowledge, and rewrites noun compounds by matching $M H$ ("American musicians") to queries of the form "H(.*)M" ( "musicians from America").

\section{$4.2 \quad$ Inputs}

We assume two inputs: 1) an IsA repository, $\mathcal{O}$, containing $\langle e, C\rangle$ tuples where $C$ is a category and $e$ is an instance of $C$, and 2) a fact repository, $\mathcal{D}$, containing $\langle s, p, o, w\rangle$ tuples where $s$ and $o$ are noun phrases, $p$ is a predicate, and $w$ is a confidence that $p$ expresses a true relation between $s$ and $o$. Both $\mathcal{O}$ and $\mathcal{D}$ are extracted from a sample of around 1 billion Web documents in English. The supplementary material gives additional details.

We instantiate $\mathcal{O}$ with an IsA repository constructed by applying Hearst patterns to the Web documents. Instances are represented as automatically-disambiguated entity mentions ${ }^{4}$ which, when possible, are resolved to Wikipedia pages. Classes are represented as (non-disambiguated) natural language strings. We instantiate $\mathcal{D}$ with a large repository of facts extracted using in-house implementations of ReVerb (Fader et al., 2011) and OLLIE (Mausam et al., 2012). The predicates are extracted as natural language strings. Subjects and objects may be either disambiguated entity references or natural language strings. Every tuple is included in both the forward and the reverse direction. E.g. 〈jazz, perform at, venue〉 also appears as $\langle$ venue, $\leftarrow$ perform at, jazz $\rangle$, where $\leftarrow$ is a spe-

\footnotetext{
4 "Entity mentions" may be individuals, like "Barack Obama", but may also be concepts like "jazz".
}

cial character signifying inverted predicates. These inverted predicates simplify the following definitions. In total, $\mathcal{O}$ contains $1.1 \mathrm{M}$ tuples and $\mathcal{D}$ contains $30 \mathrm{M}$ tuples.

\subsection{Building Property Profiles}

Properties. Let $I$ be a function that takes as input a noun phrase $M H$ and returns a property profile for $M$ relative to $H$. We define a "property" to be a tuple of a subject, predicate and object in which the subject position $^{5}$ is a wildcard, e.g. $\langle *$, born in, America $\rangle$. Any instance that fills the wildcard slot then "has" the property. We expand adjectival modifiers to encompass nominalized forms using a nominalization dictionary extracted from WordNet (Miller, 1995). If $M H$ is "American musician" and we require a tuple to have the form $\langle H, p, M, w\rangle$, we will include tuples in which the third element is either "American" or "America".

Relating $M$ to $H$ Directly. We first build property profiles by taking the predicate and object from any tuple in $\mathcal{D}$ in which the subject is the head and the object is the modifier:

$$
I_{1}(M H)=\{\langle\langle p, M\rangle, w\rangle \mid\langle H, p, M, w\rangle \in \mathcal{D}\}
$$

Relating $M$ to an Instance of $H$. We also consider an extension in which, rather than requiring the subject to be the class label $H$, we require the subject to be an instance of $H$.

$$
\begin{array}{r}
I_{2}(M H)=\{\langle\langle p, M\rangle, w\rangle \mid\langle e, H\rangle \in \mathcal{O} \\
\wedge\langle e, p, M, w\rangle \in \mathcal{D}\}
\end{array}
$$

Modifier Expansion. In practice, when building property profiles, we do not require that the object of the fact tuple match the modifier exactly, as suggested in Eq. 4 and 5. Instead, we follow Paşca (2015) and take advantage of facts involving distributionally similar modifiers. Specifically, rather than looking only at tuples in $\mathcal{D}$ in which the object matches $M$, we consider all tuples, but discount the weight proportionally to the similarity between $M$ and the object of the tuple.

\footnotetext{
${ }^{5}$ Inverse predicates capture properties in which the wildcard is conceptually the object of the relation, but occupies the subject slot in the tuple. For example, $\langle$ venue, $\leftarrow$ perform at, jazz〉 captures that a "jazz venue" is a "venue" e such that "jazz performed at $e$ ".
} 


\begin{tabular}{|l|l|l|l||l|l|}
\hline \multicolumn{4}{|c|}{ Good Property Profiles } & \multicolumn{2}{|c|}{ Bad Property Profiles } \\
\hline rice dish & French violinist & Led Zeppelin song & still life painter & child actor & risk manager \\
\hline * serve with rice & $*$ live in France & Led Zeppelin write $*$ & $*$ known for still life & $*$ have child & $*$ take risk \\
$*$ include rice & $*$ born in France & Led Zeppelin play $*$ & $*$ paint still life & $*$ expect child \\
$*$ be at risk & $*$ play child & $*$ be aware of risk \\
\hline consist of rice & $*$ speak French & Led Zeppelin have $*$ & still life be by $*$ & $*$ play \\
\hline
\end{tabular}

Table 1: Example property profiles learned by observing predicates that relate instances of class $H$ to modifier $M\left(I_{2}\right)$. Results are similar when using the class label $H$ directly $\left(I_{1}\right)$. We spell out inverted predicates (Section 4.2) so wildcards (*) may appear as subjects or objects.

Thus, $I_{1}$ is computed as below:

$$
\begin{array}{r}
I_{1}(M H)=\{\langle\langle p, M\rangle, w \times \operatorname{sim}(M, N)\rangle \\
\mid\langle H, p, N, w\rangle \in \mathcal{D}\}
\end{array}
$$

where $\operatorname{sim}(M, N)$ is the cosine similarity between $M$ and $N$. $I_{2}$ is computed analogously. We compute sim using a vector space built from Web documents following Lin and $\mathrm{Wu}$ (2009); Pantel et al. (2009). We retain the 100 most similar phrases for each of $\sim 10 \mathrm{M}$ phrases, and consider all other similarities to be 0 .

\subsection{Analysis of Property Profiles}

Table 1 provides examples of good and bad property profiles for several $\mathrm{MH}$ s. In general, frequent relations between $M$ and $H$ capture relevant properties of $M H$, but it is not always the case. To illustrate, the most frequently discussed relation between "child" and "actor" is that actors have children, but this property is not indicative of the meaning of "child actor". Qualitatively, the top-ranked interpretations learned by using the head noun directly $\left(I_{1}\right.$, Eq. 4) are very similar to those learned using instances of the head $\left(I_{2}\right.$, Eq. 5$)$. However, $I_{2}$ returns many more properties (10 on average per $M H$ ) than $I_{1}$ (just over 1 on average). Anecdotally, we see that $I_{2}$ captures more specific relations than does $I_{1}$. For example, for "jazz musicians", both methods return "* write jazz" and "* compose jazz", but $I_{2}$ additionally returns properties like "* be $m a$ jor creative influence in jazz". We compare $I_{1}$ and $I_{2}$ quantitatively in Section 6. Importantly, we do see that both $I_{1}$ and $I_{2}$ are capable of learning head-specific property profiles for a modifier. Table 2 provides examples.

\section{Class-Instance Identification}

Instance finding. After finding properties that relate a modifier to a head, we turn to the task of identifying instances of fine-grained

\begin{tabular}{|l|l|}
\hline Class Label & Property Profile \\
\hline American company & $*$ based in America \\
\hline American composer & $*$ born in America \\
\hline American novel & $*$ written in America \\
\hline$\underline{\text { jazz album }}$ & $*$ features jazz \\
\hline jazz composer & $*$ writes jazz \\
jazz venue & jazz performed at $*$ \\
\hline
\end{tabular}

Table 2: Head-specific property profiles learned by relating instances of $H$ to the modifier $M\left(I_{2}\right)$. Results are similar using $I_{1}$.

classes. That is, for a given modifier $M$, we want to instantiate the function $\phi_{M}$ from Eq. 3. In practice, rather than being a binary function that decides whether or not $e$ is in class $M H$, our instantiation, $\hat{\phi}_{M}$, will return a realvalued score expressing the confidence that $e$ is a member of $M H$. For notational convenience, let $\mathcal{D}(\langle s, p, o\rangle)=w$, if $\langle s, p, o, w\rangle \in \mathcal{D}$ and 0 otherwise. We define $\hat{\phi}_{M}$ as follows:

$$
\hat{\phi}_{M}(H, e)=\sum_{\langle\langle p, o\rangle, \omega\rangle \in I(M H)} \omega \times \mathcal{D}(\langle e, p, o\rangle)
$$

Applying $M$ to $H$, then, is as in Eq. 3 except that instead of a discrete set, it returns a scored list of candidate instances:

$$
\llbracket M \rrbracket(H)=\left\{\left\langle e, \hat{\phi}_{M}(H, e)\right\rangle \mid\langle e, H\rangle \in \mathcal{O}\right\}
$$

Ultimately, we need to identify instances of arbitrary class labels, which may contain multiple modifiers. Given a class label $C=$ $M_{1} \ldots M_{k} H$ that contains a head $H$ preceded by modifiers $M_{1} \ldots M_{k}$, we generate a list of candidate instances by finding all instances of $H$ that have some property to support every modifier:

$$
\bigcap_{i=1}^{k}\left\{\langle e, s(e)\rangle \mid\langle e, w\rangle \in \llbracket M_{i} \rrbracket(H) \wedge w>0\right\}
$$


where $s(e)$ is the mean ${ }^{6}$ of the scores assigned by each separate $\hat{\phi}_{M_{i}}$. From here on, we use Mods to refer to our method that generates lists of instances for a class using Eq. 8 and 9. When $\hat{\phi}_{M}$ (Eq. 7) is implemented using $I_{1}$, we use the name $\mathbf{M o d s}_{H}$ (for "heads"). When it is implemented using $I_{2}$, we use the

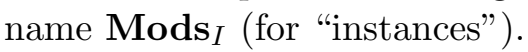

Weakly Supervised Reranking. Eq. 8 uses a naive ranking in which the weight for $e \in M H$ is the product of how often $e$ has been observed with some property and the weight of that property for the class $M H$. Thus, instances of $H$ with overall higher counts in $\mathcal{D}$ receive high weights for every $M H$. We therefore train a simple logistic regression model to predict the likelihood that $e$ belongs to $M H$. We use a small set of features ${ }^{7}$, including the raw weight as computed in Eq. 7. For training, we sample $\langle e, C\rangle$ pairs from our IsA repository $\mathcal{O}$ as positive examples and random pairs that were not extracted by any Hearst pattern as negative examples. We frame the task as a binary prediction of whether $e \in C$, and use the model's confidence as the value of $\hat{\phi}_{M}$ in place of the function in Eq. 7 .

\section{Evaluation}

\subsection{Experimental Setup}

Evaluation Sets. We evaluate our models on their ability to return correct instances for arbitrary class labels. As a source of evaluation data, we use Wikipedia category pages (e.g., http://en.wikipedia.org/wiki/Category: Pakistani_film_actresses). These are pages in which the title is the name of the category ("pakistani film actresses") and the body is a manually curated list of links to other pages that fall under the category. We measure the precision and recall of each method for discovering the instances listed on these pages given the page title (henceforth "class label").

We collect the titles of all Wikipedia category pages, removing those in which the last word is capitalized or which contain fewer than three words. These heuristics are intended to retain compositional titles in which the head is a single common noun. We also remove

\footnotetext{
${ }^{6}$ Also tried minimum, but mean gave better results.

${ }^{7}$ Feature templates in supplementary material.
}

Evaluation Set: Examples of Class Labels

UniformSet: 2008 california wildfires · australian army chaplains · australian boy bands · canadian military nurses · canberra urban places · cellular automaton rules . chinese rice dishes - coldplay concert tours · daniel libeskind designs - economic stimulus programs · german film critics · invasive amphibian species · latin political phrases · log flume rides - malayalam short stories - pakistani film actresses - puerto rican sculptors $\cdot$ string theory books

WeightedSet: ancient greek physicists · art deco sculptors · audio engineering schools - ballet training methods · bally pinball machines · british rhythmic gymnasts · calgary flames owners · canadian rock climbers · canon l-series lenses · emi classics artists $\cdot$ free password managers $\cdot$ georgetown university publications - grapefruit league venues . liz claiborne subsidiaries · miss usa 2000 delegates - new zealand illustrators · russian art critics

Table 3: Examples of class labels from evaluation sets.

any titles that contain links to sub-categories. This is to favor fine-grained classes ( "pakistani film actresses") over coarse-grained ones ( "film actresses"). We perform heuristic modifier chunking in order to group together multiword modifiers (e.g., "puerto rican"); for details, see supplementary material. From the resulting list of class labels, we draw two samples of 100 labels each, enforcing that no $H$ appear as the head of more than three class labels per sample. The first sample is chosen uniformly at random (denoted UniformSet). The second (WeightedSet) is weighted so that the probability of drawing $M_{1} \ldots M_{k} H$ is proportional to the total number of class labels in which $H$ appears as the head. These different evaluation sets ${ }^{8}$ are intended to evaluate performance on the head versus the tail of class label distribution, since information retrieval methods often perform differently on different parts of the distribution. On average, there are 17 instances per category in UniformSet and 19 in WeightedSet. Table 3 gives examples of class labels.

Baselines. We implement two baselines using our IsA repository $(\mathcal{O}$ as defined in Section 4.1). Our simplest baseline ignores modifiers altogether, and simply assumes that any instance of $H$ is an instance of $M H$, regardless of $M$. In this case the confidence value for

\footnotetext{
${ }^{8}$ Available at http://www.seas.upenn.edu/ nlp/ resources/finegrained-class-eval.gz
} 
$\langle e, M H\rangle$ is equivalent to that for $\langle e, H\rangle$. We refer to this baseline simply as Baseline. Our second, stronger baseline uses the IsA repository directly to identify instances of the finegrained class $C=M_{1} \ldots M_{k} H$. That is, we consider $e$ to be an instance of the class if $\langle e, C\rangle \in \mathcal{O}$, meaning the entire class label appeared in a source sentence matching some Hearst pattern. We refer to this baseline as Hearst. The weight used to rank the candidate instances is the confidence value assigned by the Hearst pattern extraction (Section 4.2).

Compositional Models. As a baseline compositional model, we augment the Hearst baseline via set intersection. Specifically, for a class $C=M_{1} \ldots M_{k} H$, if each of the $M_{i} H$ appears in $\mathcal{O}$ independently, we take the instances of $C$ to be the intersection of the instances of each of the $M_{i} H$. We assign the weight of an instance $e$ to be the sum of the weights associated with each independent modifier. We refer to this method as Hearst $\cap$. It is roughly equivalent to (Paşca, 2014). We contrast it with our proposed model, which recognizes instances of a fine-grained class by 1) assigning a meaning to each modifier in the form of a property profile and 2) checking whether a candidate instance exhibits these properties. We refer to the versions of our method as Mods $\mathbf{S}_{H}$ and $\operatorname{Mods}_{I}$, as described in Section 5. When relevant, we use "raw" to refer to the version in which instances are ranked using raw weights and "RR" to refer to the version in which instances are ranked using logistic regression (Section 5). We also try using the proposed methods to extend rather than replace the Hearst baseline. We combine predictions by merging the ranked lists produced by each system: i.e. the score of an instance is the inverse of the sum of its ranks in each of the input lists. If an instance does not appear at all in an input list, its rank in that list is set to a large constant value. We refer to these combination systems as Hearst+Mods ${ }_{H}$ and Hearst+Mods . $_{I}$.

\subsection{Results}

Precision and Coverage. We first compare the methods in terms of their coverage, the number of class labels for which the method is able to find some instance, and their precision, to what extent the method is able to correctly rank true instances of the class above non-instances. We report total coverage, the number of labels for which the method returns any instance, and correct coverage, the number of labels for which the method returns a correct instance. For precision, we compute the average precision (AP) for each class label. AP ranges from 0 to 1 , where 1 indicates that all positive instances were ranked above all negative instances. We report mean average precision (MAP), which is the mean of the APs across all the class labels. MAP is only computed over class labels for which the method returns something, meaning methods are not punished for returning empty lists.

Table 4 gives examples of instances returned for several class labels and Table 5 shows the precision and coverage for each of the methods. Figure 2 illustrates how the single mean AP score (as reported in Table 5) can misrepresent the relative precision of different methods. In combination, Table 5 and Figure 2 demonstrate that the proposed methods extract instances about as well as the baseline, whenever the baseline can extract anything at all; i.e. the proposed method does not cause a precision drop on classes covered by the baseline. In addition, there are many classes for which the baseline is not able to extract any instances, but the proposed method is. None of the methods can extract some of the gold instances, such as "Dictator perpetuo" and "Furor Teutonicus" of the gold class "latin political phrases".

Table 5 also reveals that the reranking model (RR) consistently increases MAP for the proposed methods. Therefore, going forward, we only report results using the reranking model (i.e. $\operatorname{Mods}_{H}$ and Mods ${ }_{I}$ will refer to $\operatorname{Mods}_{H} \mathrm{RR}$ and $\operatorname{Mods}_{I} \mathrm{RR}$, respectively).

Manual Re-Annotation. It possible that true instances of a class are missing from our Wikipedia reference set, and thus that our precision scores underestimate the actual precision of the systems. We therefore manually verify the top 10 predictions of each of the systems for a random sample of 25 class labels. We choose class labels for which Hearst was able to return at least one instance, in order to ensure reliable precision 
Flemish still life painters: Clara Peeters · Willem Kalf · Jan Davidsz de Heem · Pieter Claesz · Peter Paul Rubens · Frans Snyders · Jan Brueghel the Elder · Hans Memling • Pieter Bruegel the Elder · Caravaggio · Abraham Brueghel

Pakistani cricket captains: Salman Butt · Shahid Afridi · Javed Miandad · Azhar Ali · Greg Chappell · Younis Khan · Wasim Akram · Imran Khan · Mohammad Hafeez · Rameez Raja · Abdul Hafeez Kardar · Waqar Younis · Sarfraz Ahmed

Thai buddhist temples: Wat Buddhapadipa - Wat Chayamangkalaram · Wat Mongkolratanaram - Angkor Wat · Preah Vihear Temple · Wat Phra Kaew · Wat Rong Khun · Wat Mahathat Yuwaratrangsarit - Vat Phou - Tiger Temple - Sanctuary of Truth - Wat Chalong · Swayambhunath · Mahabodhi Temple · Tiger Cave Temple $\cdot$ Harmandir Sahib

Table 4: Instances extracted for several fine-grained classes from Wikipedia. Lists shown are from $\operatorname{Mods}_{I}$. Instances in italics were also returned by Hearst $\cap$. Strikethrough denotes incorrect.

\begin{tabular}{|lcccc|}
\hline & \multicolumn{2}{c}{ UniformSet } & \multicolumn{2}{c|}{ WeightedSet } \\
& Coverage & MAP & Coverage & MAP \\
\hline Baseline & $95 / 70$ & 0.01 & $98 / 74$ & 0.01 \\
Hearst & $9 / 9$ & 0.63 & $8 / 8$ & 0.80 \\
Hearst $\cap$ & $13 / 12$ & 0.62 & $9 / 9$ & 0.80 \\
\hline Mods $_{H}$ raw & $56 / 32$ & 0.23 & $50 / 30$ & 0.16 \\
Mods $_{H}$ RR & $56 / 32$ & 0.29 & $50 / 30$ & 0.25 \\
Mods $_{I}$ raw & $62 / 36$ & 0.18 & $59 / 38$ & 0.20 \\
Mods $_{I}$ RR & $62 / 36$ & 0.24 & $59 / 38$ & 0.23 \\
\hline
\end{tabular}

Table 5: Coverage and precision for populating Wikipedia category pages with instances. "Coverage" is the number of class labels (out of 100) for which at least one instance was returned, followed by the number for which at least one correct instance was returned. "MAP" is mean average precision. MAP does not punish methods for returning empty lists, thus favoring the baseline (see Figure 2).

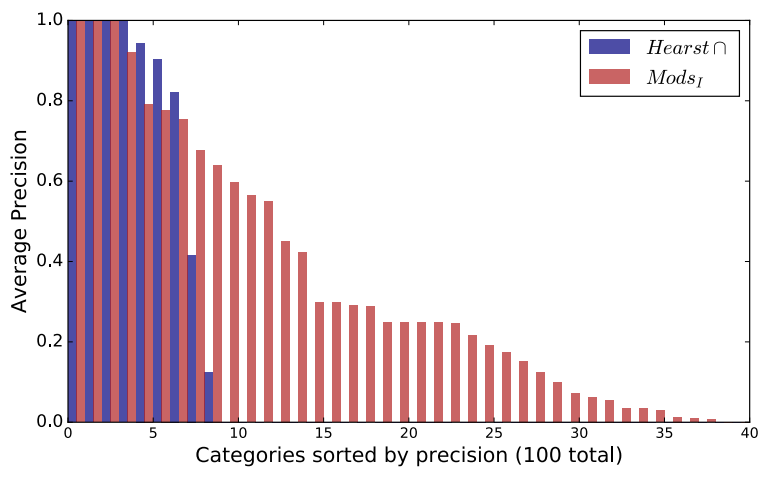

Figure 2: Distribution of AP over 100 class labels in WeightedSet. The proposed method (red) and the baseline method (blue) achieve high AP for the same number of classes, but

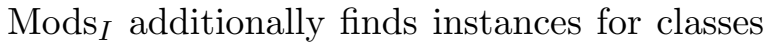
for which the baseline returns nothing.

estimates. For each of these labels, we manually check the top 10 instances proposed by each method to determine whether each belongs to the class. Table 6 shows the precision scores for each method computed against the original Wikipedia list of instances and against our manually-augmented list of gold instances. The overall ordering of the systems does not change, but the precision scores increase notably after re-annotation. We continue to evaluate against the Wikipedia lists, but acknowledge that reported precision is likely an underestimate of true precision.

\begin{tabular}{|lcc|}
\hline & Wikipedia & Gold \\
\hline Hearst & 0.56 & 0.79 \\
Hearst $\cap$ & 0.53 & 0.78 \\
\hline Mods $_{H}$ & 0.23 & 0.39 \\
Mods $_{I}$ & 0.24 & 0.42 \\
Hearst+Mods $_{H}$ & 0.43 & 0.63 \\
Hearst+Mods $_{I}$ & 0.43 & 0.63 \\
\hline
\end{tabular}

Table 6: P@10 before vs. after re-annotation; Wikipedia underestimates true precision.

\begin{tabular}{|lcccc|}
\hline & \multicolumn{2}{c|}{ UniformSet } & \multicolumn{2}{c|}{ WeightedSet } \\
& AUC & Recall & AUC & Recall \\
\hline Baseline & 0.55 & 0.23 & 0.53 & 0.28 \\
Hearst & 0.56 & 0.03 & 0.52 & 0.02 \\
Hearst $\cap$ & 0.57 & 0.04 & 0.53 & 0.02 \\
\hline Mods $_{H}$ & 0.68 & 0.08 & 0.60 & 0.06 \\
Mods $_{I}$ & 0.71 & 0.09 & 0.65 & 0.09 \\
Hearst $\cap+$ Mods $_{H}$ & 0.70 & 0.09 & 0.61 & 0.08 \\
Hearst $\cap+$ Mods $_{I}$ & 0.73 & 0.10 & 0.66 & 0.10 \\
\hline
\end{tabular}

Table 7: Recall of instances on Wikipedia category pages, measured against the full set of instances from all pages in sample. AUC captures tradeoff between true and false positives. 


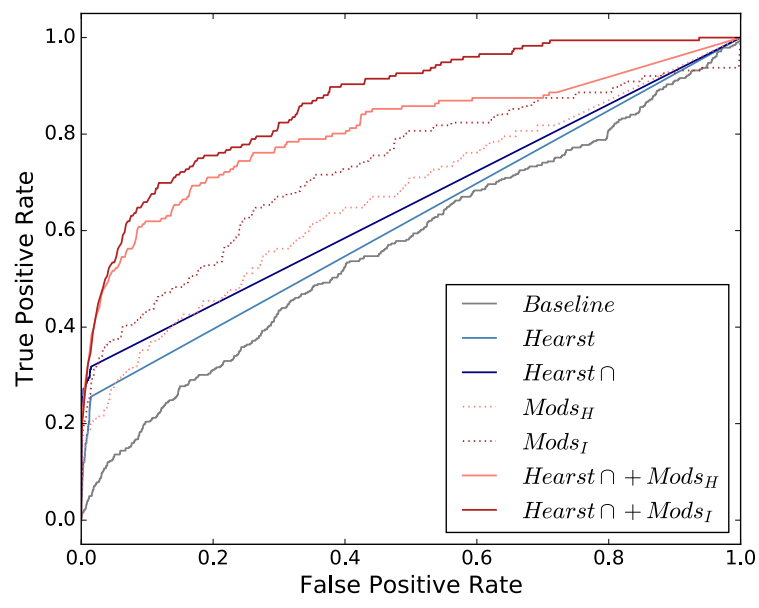

(a) Uniform random sample (UniformSet).

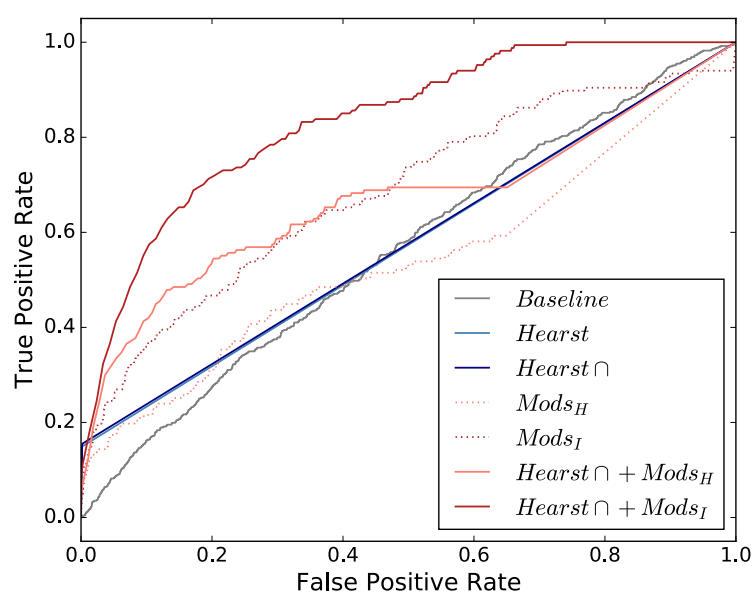

(b) Weighted random sample (WeightedSet).

Figure 3: ROC curves for selected methods (Hearst in blue, proposed in red). Given a ranked list of instances, ROC curves plot true positives vs. false positives retained by setting various cutoffs. The curve becomes linear once all remaining instances have the same score (e.g., 0), as this makes it impossible to add true positives without also including all remaining false positives.

Precision-Recall Analysis. We next look at the precision-recall tradeoff in terms of the area under the curve (AUC) when each method attempts to rank the complete list of candidate instances. We take the union of all of the instances proposed by all of the methods (including the Baseline method which, given a class label $M_{0} \ldots M_{k} H$, proposes every instance of the head $H$ as a candidate). Then, for each method, we rank this full set of candidates such that any instance returned by the method is given the score the method assigns, and every other instance is scored as 0 . Table 7 reports the AUC and recall. Figure 3 plots the full ROC curves. The requirement by Hearst that class labels appear in full in a single sentence results in very low recall, which translates into very low AUC when considering the full set of candidate instances. In comparison, the proposed compositional methods make use of a larger set of sentences, and provide nonzero scores for many more candidates, resulting in a $>10$ point increase in AUC on both UniformSet and WeightedSet (Table 7).

\section{Conclusion}

We have presented an approach to IsA extraction that takes advantage of the compositionality of natural language. Existing approaches often treat class labels as atomic units that must be observed in full in order to be pop- ulated with instances. As a result, current methods are not able to handle the infinite number of classes describable in natural language, most of which never appear in text. Our method reasons about each modifier in the label individually, in terms of the properties that it implies about the instances. This approach allows us to harness information that is spread across multiple sentences, significantly increasing the number of fine-grained classes that we are able to populate.

\section{Acknowledgments}

The paper incorporates suggestions on an earlier version from Susanne Riehemann. Ryan Doherty offered support in refining and accessing the fact repository used in the evaluation.

\section{References}

M. Bansal, D. Burkett, G. de Melo, and D. Klein. 2014. Structured learning for taxonomy induction with belief propagation. In Proceedings of the 52nd Annual Meeting of the Association for Computational Linguistics (ACL-14). Baltimore, Maryland, pages 1041-1051.

M. Baroni and R. Zamparelli. 2010. Nouns are vectors, adjectives are matrices: Representing adjective-noun constructions in semantic space. In Proceedings of the 2010 Conference on Empirical Methods in Natural Language Processing (EMNLP-10). Cambridge, Massachusetts, pages 1183-1193. 
C. Bizer, J. Lehmann, G. Kobilarov, S. Auer, C. Becker, R. Cyganiak, and S. Hellmann. 2009. DBpedia - a crystallization point for the Web of data. Journal of Web Semantics 7(3):154-165.

E. Choi, T. Kwiatkowski, and L. Zettlemoyer. 2015. Scalable semantic parsing with partial ontologies. In Proceedings of the 53rd Annual Meeting of the Association for Computational Linguistics (ACL-15). Beijing, China, pages 1311-1320.

B. Dalvi, W. Cohen, and J. Callan. 2012. Websets: Extracting sets of entities from the Web using unsupervised information extraction. In Proceedings of the 5th ACM Conference on Web Search and Data Mining (WSDM-12). Seattle, Washington, pages 243-252.

G. de Melo and G. Weikum. 2010. MENTA: Inducing multilingual taxonomies from Wikipedia. In Proceedings of the 19th International Conference on Information and Knowledge Management (CIKM-10). Toronto, Canada, pages 1099-1108.

A. Fader, S. Soderland, and O. Etzioni. 2011. Identifying relations for open information extraction. In Proceedings of the 2011 Conference on Empirical Methods in Natural Language Processing (EMNLP-11). Edinburgh, Scotland, pages 1535-1545.

M. Fares, S. Oepen, and E. Velldal. 2015. Identifying compounds: On the role of syntax. In International Workshop on Treebanks and Linguistic Theories (TLT-14). Warsaw, Poland, pages 273-283.

T. Flati, D. Vannella, T. Pasini, and R. Navigli. 2014. Two is bigger (and better) than one: the Wikipedia Bitaxonomy project. In Proceedings of the 52nd Annual Meeting of the Association for Computational Linguistics (ACL-14). Baltimore, Maryland, pages 945-955.

M. Hearst. 1992. Automatic acquisition of hyponyms from large text corpora. In Proceedings of the 14th Conference on Computational Linguistics (COLING-92). pages 539-545.

I. Heim and A. Kratzer. 1998. Semantics in Generative Grammar, volume 13. Blackwell Oxford.

I. Hendrickx, Z. Kozareva, P. Nakov, D. Ó Séaghdha, S. Szpakowicz, and T. Veale. 2013. SemEval-2013 task 4: Free paraphrases of noun compounds. In Proceedings of Proceedings of the 7th International Workshop on Semantic Evaluation (SemEval-13). pages 138-143.

J. Hoffart, F. Suchanek, K. Berberich, and G. Weikum. 2013. YAGO2: a spatially and temporally enhanced knowledge base from Wikipedia. Artificial Intelligence Journal. Special Issue on Artificial Intelligence, Wikipedia and Semi-Structured Resources 194:28-61.
H. Kamp and B. Partee. 1995. Prototype theory and compositionality. Cognition 57(2):129-191.

N. Kim and P. Nakov. 2011. Large-scale noun compound interpretation using bootstrapping and the Web as a corpus. In Proceedings of the 2011 Conference on Empirical Methods in Natural Language Processing (EMNLP-11). Edinburgh, Scotland, pages 648-658.

J. Kirschnick, H. Hemsen, and V. Markl. 2016. Jedi: Joint entity and relation detection using type inference. In Proceedings of the 54 th $A n$ nual Meeting of the Association for Computational Linguistics (ACL-16) - System Demonstrations. Berlin, Germany, pages 61-66.

Z. Kozareva and E. Hovy. 2010. A semi-supervised method to learn and construct taxonomies using the web. In Proceedings of the 2010 Conference on Empirical Methods in Natural Language Processing (EMNLP-10). Cambridge, Massachusetts, pages 1110-1118.

D. Lin and X. Wu. 2009. Phrase clustering for discriminative learning. In Proceedings of the $47 \mathrm{th}$ Annual Meeting of the Association for Computational Linguistics (ACL-IJCNLP-09). Singapore, pages 1030-1038.

Mausam, M. Schmitz, S. Soderland, R. Bart, and O. Etzioni. 2012. Open language learning for information extraction. In Proceedings of the 2012 Joint Conference on Empirical Methods in Natural Language Processing and Computational Natural Language Learning (EMNLPCoNLL-12). Jeju Island, Korea, pages 523-534.

G. Miller. 1995. WordNet: a lexical database. Communications of the ACM 38(11):39-41.

D. Movshovitz-Attias and W. Cohen. 2015. Kblda: Jointly learning a knowledge base of hierarchy, relations, and facts. In Proceedings of the 53rd Annual Meeting of the Association for Computational Linguistics and the 7th International Joint Conference on Natural Language Processing (ACL-IJCNLP-15). Beijing, China, pages 1449-1459.

P. Nakov and M. Hearst. 2013. Semantic interpretation of noun compounds using verbal and other paraphrases. ACM Transactions on Speech and Language Processing 10(3):1-51.

V. Nastase and M. Strube. 2013. Transforming Wikipedia into a large scale multilingual concept network. Artificial Intelligence 194:62-85.

P. Nulty and F. Costello. 2013. General and specific paraphrases of semantic relations between nouns. Natural Language Engineering 19(03):357-384. 
D. Ó Séaghdha and A. Copestake. 2007. Cooccurrence contexts for noun compound interpretation. In Proceedings of the Workshop on a Broader Perspective on Multiword Expressions. Prague, Czech Republic, pages 57-64.

M. Paşca. 2014. Acquisition of open-domain classes via intersective semantics. In Proceedings of the 23rd World Wide Web Conference (WWW-14). Seoul, Korea, pages 551-562.

M. Paşca. 2015. Interpreting compound noun phrases using web search queries. In Proceedings of the 2015 Conference of the North American Chapter of the Association for Computational Linguistics: Human Language Technologies (NAACL-HLT-15). Denver, Colorado, pages $335-344$.

P. Pantel, E. Crestan, A. Borkovsky, A. Popescu, and V. Vyas. 2009. Web-scale distributional similarity and entity set expansion. In Proceedings of the 2009 Conference on Empirical Methods in Natural Language Processing (EMNLP09). Singapore, pages 938-947.

P. Pasupat and P. Liang. 2014. Zero-shot entity extraction from Web pages. In Proceedings of the 52nd Annual Meeting of the Association for Computational Linguistics (ACL-14). Baltimore, Maryland, pages 391-401.

S. Ponzetto and R. Navigli. 2009. Large-scale taxonomy mapping for restructuring and integrating Wikipedia. In Proceedings of the 21st International Joint Conference on Artificial Intelligence (IJCAI-09). Pasadena, California, pages 2083-2088.

S. Ponzetto and M. Strube. 2007. Deriving a large scale taxonomy from Wikipedia. In Proceedings of the 22nd National Conference on Artificial Intelligence (AAAI-07). Vancouver, British Columbia, pages 1440-1447.

S. Riedel, L. Yao, A. McCallum, and B. Marlin. 2013. Relation extraction with matrix factorization and universal schemas. In Proceedings of the 2013 Conference of the North American Association for Computational Linguistics (NAACL-HLT-13). Atlanta, Georgia, pages 7484.

V. Shwartz, Y. Goldberg, and I. Dagan. 2016. Improving hypernymy detection with an integrated path-based and distributional method. In Proceedings of the 54th Annual Meeting of the Association for Computational Linguistics ( $A C L$ 16). Berlin, Germany, pages 2389-2398.

R. Snow, D. Jurafsky, and A. Ng. 2006. Semantic taxonomy induction from heterogenous evidence. In Proceedings of the 21st International Conference on Computational Linguistics and 44th Annual Meeting of the Association for
Computational Linguistics (COLING-ACL-06). Sydney, Australia, pages 801-808.

N. Surtani and S. Paul. 2015. A vsm-based statistical model for the semantic relation interpretation of noun-modifier pairs. Proceedings of the International Conference on Recent Advances in Natural Language Processing (RANLP-15) pages $636-645$.

S. Tratz and E. Hovy. 2010. A taxonomy, dataset, and classifier for automatic noun compound interpretation. In Proceedings of the 48th Annual Meeting of the Association for Computational Linguistics (ACL-10). Uppsala, Sweden, pages 678-687.

T. Van de Cruys, S. Afantenos, and P. Muller. 2013. MELODI: A supervised distributional approach for free paraphrasing of noun compounds. In Proceedings of the Yth International Workshop on Semantic Evaluation (SemEval13). Atlanta, Georgia, pages 144-147.

P. Verga, A. Neelakantan, and A. McCallum. 2017. Generalizing to unseen entities and entity pairs with row-less universal schema. In Proceedings of the 15th Conference of the European Chapter of the Association for Computational Linguistics (EACL-17). Valencia, Spain, pages 613-622.

R. Wang and W. Cohen. 2009. Automatic set instance extraction using the Web. In Proceedings of the 47th Annual Meeting of the Association for Computational Linguistics (ACL-IJCNLP09). Singapore, pages 441-449.

D. Weiskopf. 2007. Compound nominals, context, and compositionality. Synthese 156(1):161-204.

D. Wijaya and P. Gianfortoni. 2011. Nut case: What does it mean?: Understanding semantic relationship between nouns in noun compounds through paraphrasing and ranking the paraphrases. In Proceedings of the 1st International Workshop on Search and Mining EntityRelationship Data (SMER-11). Glasgow, United Kingdom, pages 9-14.

C. Xavier and V. Strube de Lima. 2014. Boosting open information extraction with noun-based relations. In Proceedings of the 9th International Conference on Language Resources and Evaluation (LREC-14). Reykjavik, Iceland, pages 96100.

P. Young, A. Lai, M. Hodosh, and J. Hockenmaier. 2014. From image descriptions to visual denotations: New similarity metrics for semantic inference over event descriptions. Transactions of the Association for Computational Linguistics (TACL) 2:67-78. 